\title{
FACTORES PSICOSOCIALES LABORALES INMERSOS EN EL CONTEXTO DE LOS DOCENTES EN UNA INSTITUCIÓN EDUCATIVA ESTATAL DE SAN JUAN DE PASTO
}

\author{
PSYCHOSOCIAL FACTORS IMMERSED IN THE CONTEXT OF \\ EDUCATORS IN A STATE EDUCATIONAL INSTITUTION OF SAN \\ JUAN DE PASTO
}

\section{FATORES PSICOSSOCIAS DE TRABALHO IMERSO NO CONTEXTO DE PROFESSORES DE UMA INSTITUIÇÃO EDUCACIONAL DE ESTADO DE SAN JUAN DE PASTO}
DÁVILA BURBANO_Hernán Darío, LOMBANA MORA_Lady Dayanne, MATABANCHOY TULCAN_Sonia Maritza, ZAMBRANO GUERRERO_ Christian Alexander

Psicólogo, Universidad de Nariño. Email: dardodavila@gmail.com, Colombia Psicólogo, Universidad de Nariño. Email: lady08.1994@gmail.com, Colombia.

$\mathrm{PhD}$ (c) Psicología. Magíster Gerencia del Talento Humano. Docente Universidad de Nariño, investigadora del Grupo COGNICED.Pasto, Colombia.e-mail: somapsicologa@, yahoo.es, Colombia.

Magíster en Educación desde la diversidad. Docente del Programa de Psicología, Universidad de Nariño, investigador del Grupo Psicología y Salud. Pasto, Colombia. e-mail: christianzambris@hotmail.com, Colombia.

Recibido: 06 de julio de 2017

Aprobación definitiva: 10 de junio de 2018

\section{DOI: http://dx.doi.org/10.22267/rtend.181902.101}

\section{RESUMEN}

Los factores psicosociales deben representar un papel importante dentro de cada organización ya que estos afectan directamente el desempeño del trabajador y su salud, como también la funcionalidad de la empresa, es por ello que el presente estudio tiene como objetivo la identificación de los factores psicosociales 
intralaborales y extralaborales en docentes de una Institución Educativa Estatal de San Juan de Pasto, a través de una metodología descriptiva con diseño transversal, donde se aplicó tres cuestionarios del Ministerio de la Protección Social de Colombia (2010): Batería de Instrumentos para la Evaluación de Factores de Riesgo Psicosocial (Ficha de Datos Generales, el Cuestionario de Riesgo Psicosocial Intralaboral (forma A) y el Cuestionario de Riesgo Psicosocial Extralaboral). Los resultados muestran que los docentes no están exentos de estar expuestos en riesgos intralaborales y extralaborales ya que las diferentes condiciones que se presentan dentro y fuera del lugar de trabajo, afectan su salud y desempeño en la Institución, lo cual se evidencia en la mayor parte de la población que puntúa algunas dimensiones en riesgo alto y muy alto.

Palabras clave: Factores de riesgo intralaboral, Factores de riesgo extralaboral, Docentes.

JEL: I31, J28, J81.

\begin{abstract}
The psychosocial factors should play an important role within each organization since these directly affect the worker's performance and health, as well as the functionality of the company. For this reason, the present study aims to identify the intra-labor and extra-labor psychosocial factors in educators of a State Educational Institution of San Juan de Pasto through a descriptive methodology with a cross-sectional design. In the study, three questionnaires from the Colombian Ministry of Social Protection (2010) were applied: the Battery Instruments for the Evaluation of Psychosocial Risk Factors (General data Sheet, the Intra-labor Psychosocial Risk Questionnaire (form A), and the Extra-Labor Psychosocial Risk Questionnaire). The results show that educators are not exempt from being exposed to intra-labor and extra- labor risks, since the different conditions that take place inside and outside the workplace affect their health and performance in the Institution. These findings are evidenced in most of the population that scores some dimensions at high and very high risk.
\end{abstract}

Keywords: Intra-labor risk factors, Extra-labor risk factors, Educators. JEL: I31, J28, J81. 


\section{RESUMO}

Fatores psicossociais devem desempenhar um papel importante dentro de cada organização como estas afetam diretamente o desempenho do trabalhador e sua saúde, também como a funcionalidade da empresa, razão pela qual este estudo tem como objetivo identificar os fatores psicossocias dentro do trabalho e fora do local de trabalho em profesores de uma Instituição de ensino Estadual de San Juan de Pasto, através de uma metodologia descritiva com delineamento transversal, onde foram aplicados três questionários do Ministério da Proteção social da Colômbia (2010): Instrumentos de bateria para a avaliação de fatores Risco psicossocial (Folha de dados Gerais, Risco psicossocial Questionário dentro do trabalho (forma A) e o Questionário de Risco psicossocial fora do trabalho). Os resultados mostram que os professores não estão isentos de serem expostos em riscos dentro e fora do local de trabalho condições tão diferentes que surgem dentro e fora do local de trabalho afectam a sua saúde e desempenho na instituição, que é evidente na maior parte da população que pontua algumas dimensões de risco alto e muito alto.

Palavras-chave: fatores de risco dentro do trabalho, fatores de risco fora do local de trabalho, professores.

JEL: I31, J28, J81.

\section{INTRODUCCIÓN}

En la actualidad el estudio de los factores psicosociales deben ser relevantes para cada institución, considerando que el hombre está expuesto a sobre exigirse en su desempeño, a nivel competitivo, productivo y de eficiencia a causa de las condiciones socioeconómicas en las que la persona se encuentra, estas pueden generar que se considere al trabajador como una máquina de producción, dejando de lado el componente emocional, que es parte fundamental de las personas (Enríquez, 2008: 74).

Los factores psicosociales son inherentes a las condiciones propias de los individuos, las organizaciones y el medio ambiente, y se consideran como elementos que potencialmente pueden afectar la calidad de la vida laboral de los individuos, la salud física o psíquica de las personas (Velásquez \& Bedoya, 2010: 3). Estos factores no constituyen un riesgo para el trabajador, sino hasta 
el momento en que por su frecuencia, tiempo de exposición o intensidad se convierten en nocivos para el bienestar y salud del individuo, desequilibrando su relación con el trabajo o con el entorno, convirtiéndose así en factores de riesgo psicosocial laboral (Múnera, Sáenz \& Cardona, 2013: 165).

La Organización Internacional de Trabajo [OIT], (2002), plantea que a pesar de los avances frente a la prevención de accidentes y enfermedades laborales, cada año alrededor de 2 millones de personas mueren por actividades relacionadas con el trabajo, además suceden alrededor de 250 millones de accidentes laborales y se diagnostican 160 millones de enfermedades profesionales en todo el mundo. El Consejo Colombiano de Seguridad [CCS] en el año 2015, nos revela las cifras en Colombia donde los últimos años nos muestran un incremento bastante notable ya que para el año 2009 se encontraron un total de 6 mil enfermedades laborales y en el año 2014 se encontraron 9.700 casos.

Este tipo de situaciones genera pérdidas económicas pues se ve afectada la productividad y la capacidad de trabajo, lo que a nivel mundial trae la pérdida de un $4 \%$ aproximadamente del producto interno bruto (PIB), representado en el pago de indemnizaciones, de gastos médicos, daños materiales, pérdidas de ingresos y los gastos de formación del personal de sustitución. Por ello se considera necesario que el empleador tenga una información necesaria para poder desarrollar estrategias de prevención. (Organización Mundial de Trabajo, 2002).

Herazo, Ospina \& Ramirez (2013:15), afirman que, para que una organización tenga éxito dentro del mercado actual, ésta debe valorar el talento humano del cual dispone, ya que aquellas organizaciones que gestionan y potencializan su fuerza laboral, son más efectivas. Para esto es necesario que adopten estrategias dirigidas a satisfacer las necesidades tanto de la organización como de sus trabajadores, ya que en la medida que las personas se identifiquen con la organización habrá mayor productividad, logrando que sea más humana sin tener que perder el fin único de la empresa.

Se define los factores psicosociales laborales como las interrelaciones que se pueden generar, por una parte entre el trabajo, el medio ambiente, la satisfacción en el puesto, las condiciones de organización, y por la otra, las capacidades del trabajador, sus necesidades, cultura y su situación personal fuera del trabajo (Comité Mixto Organización Internacional del Trabajo [OIT]; 
Organización Mundial de la Salud [OMS], 1984: 3). Estos factores, por medio de las percepciones y experiencias, pueden incidir en la salud, el rendimiento y la satisfacción en el trabajo (Comité Mixto Organización Internacional del Trabajo [OIT]; Organización Mundial de la Salud [OMS], 1984: 3).

De esta forma, los factores de riesgo psicosocial laboral hacen referencia a las condiciones físicas y ambientales que se generan en una situación laboral y que están relacionadas con la organización, el contenido del trabajo y la realización de la tarea y que tienen capacidad para afectar al bienestar o la salud (física, psíquica o social) del trabajador, así como al desarrollo del trabajo (Caballero et al., 2009: 143; Múnera et al., 2013: 165). La exposición a factores de riesgo psicosocial intralaboral y extralaboral, afectan al individuo por su grado de impacto en la calidad de la vida laboral, origina consecuencias fundamentalmente en el rendimiento en el trabajo, la satisfacción y la salud del trabajador (Puentes et al., 2008: 174).

Ante la presencia de factores de riesgo psicosocial en el ambiente laboral, aumenta la probabilidad de ausentismos, rotación o abandonos, que reducen la capacidad de trabajo y afectan la productividad del trabajador, se producen problemas de seguridad laboral, incrementan los accidentes de trabajo, enfermedades laborales y acoso laboral o mobbing (Caballero et al., 2009: 143; Múnera et al, 2013: 165; Puentes, et al., 2008: 174; Velásquez \& Bedoya, 2010: 3).

Estos factores de riesgo, además de afectar el ambiente laboral también producen un fuerte impacto en el bienestar del trabajador, generan alteraciones psicoemocionales como síntomas de estrés e insatisfacción laboral (Cladellas \& Badia, 2010: 305), además aumenta la probabilidad del desarrollo de síndrome de burnout (Caballero et al., 2009: 155), alteraciones del sueño y del apetito, así como enfermedades cardiovasculares, hipertensión arterial, entre otras (Gómez \& Moreno, 2010: 394). Adicionalmente, implican costos económicos para las empresas por el tiempo perdido, gastos sanitarios, incapacidades médicas, entre otros (Caballero et al., 2009: 143).

En consecuencia, el Ministerio de la Protección Social de Colombia, considerando los múltiples efectos de los factores de riesgo psicosocial en el bienestar de los trabajadores, se expide en el año 2008 la Resolución 2646 que establece disposiciones y define responsabilidades para la identificación, 
evaluación, prevención y monitoreo permanente de la exposición a factores de riesgo psicosocial en el trabajo y para la determinación y origen de patologías causadas por el estrés ocupacional. Es por ello, que durante los años 2009 y 2010 el Ministerio de la Protección Social junto con la Universidad Javeriana diseñó la Batería de Instrumentos para la Evaluación de los Factores de Riesgo Psicosocial que fue validada y estandarizada con una muestra de trabajadores afiliados al Sistema General Riesgos Profesionales (Peláez \& Cuellar, 2014: 5).

La construcción de la Batería se basa en la definición de factores psicosociales propuesta por el Ministerio de la Protección Social de Colombia (2008) en la Resolución 2646, como aquellos factores que:

Comprenden los aspectos intralaborales, los extralaborales o externos a la organización y las condiciones individuales o características intrínsecas del trabajador, los cuales en una interrelación dinámica, mediante percepciones y experiencias, influyen en la salud y el desempeño de las personas (p. 3).

Los factores psicosociales laborales tienen en cuenta aspectos intralaborales, extralaborales y condiciones individuales del trabajador. Así, la Batería permite identificar condiciones intralaborales, es decir, características internas en el trabajo y la organización que influyen en la salud y bienestar del individuo, por medio de cuatro dominios que actúan como posibles fuentes de riesgo. Dichos dominios corresponden a demandas de trabajo, control sobre el trabajo, liderazgo y relaciones sociales en el trabajo, y recompensas (Ministerio de la Protección Social, 2010: 20).

Por otra parte, las condiciones extralaborales comprenden aspectos del entorno familiar, social y económico del trabajador, las dimensiones que evalúa el cuestionario son: tiempo fuera del trabajo, relaciones familiares, comunicación y relaciones interpersonales, situación económica del grupo familiar, características de la vivienda y su entorno, influencia del entorno extralaboral sobre el trabajo, y desplazamiento vivienda-trabajo-vivienda. (Ministerio de la Protección Social, 2010: 26)

Finalmente, las condiciones individuales aluden a las características propias de cada trabajador, características sociodemográficas y ocupacionales que pueden modular la percepción y el efecto de los factores de riesgo intralaborales y extralaborales (Ministerio de la Protección Social, 2010: 27). De esta forma, 
la Batería permite obtener información para desarrollar y focalizar programas e intervenciones dirigidas a la prevención y control de los factores de riesgo, y así promover la calidad de vida de los trabajadores (Múnera, et al., 2013: 171).

Concretamente, el sector educativo ha llegado a ser considerado uno de los más expuestos a enfermedades psicosociales, es así como Botero (2012: 119) refiere que la profesión docente está sujeta a diversos cambios que responden a las necesidades de los estudiantes, a la competitividad con la investigación, la innovación y la administración estratégica. Dichos cambios se relacionan con la implementación de nuevas tecnologías, los currículos, las estructuras organizacionales y las modalidades de contratación, que junto con otras variables como el trato con los estudiantes, el aumento en el ritmo de trabajo y las funciones, el trabajo individualizado, la edad de los docentes, sus años de experiencia y el esfuerzo por ser reconocido socialmente por su labor, crean ambientes estresantes y generan riesgos psicosociales que afecta significativamente la vida del docente (Botero 2012: 120). De igual manera Pérez (2009: 33) refiere también a la docencia como una de las profesiones con más tendencia a sufrir patologías relacionadas con los factores psicosociales, ya que la profesión docente es aquella que soporta mayor nivel de estrés, teniendo en cuenta que las diferentes demandas del medio son percibidas por el docente como excesivas o amenazantes para el bienestar e integridad, debido a que el estrés se deriva del escaso reconocimiento social, el elevado número de alumnos a los que atienden, a conflictos generados en las relaciones de la comunidad educativa y a los elevados ritmos de trabajo a los cuales se pueden ver sometidos.

De ahí, que resulte importante disminuir la exposición a riesgos psicosociales, a través de intervenciones que promuevan la salud del docente.

Diferentes investigaciones han permitido identificar que las características intrínsecas de la docencia generan situaciones que propician la prevalencia de riesgos específicos para esta población. Los resultados de diversos estudios en los que se encuentran Rodríguez, Sánchez, Dorado \& Ramírez (2014: 17) y Vélez \& Mayorga (2013: 90), en población de docentes colombianos han encontrado la presencia de ciertos factores de riesgo psicosocial entre los cuales es frecuente la presencia de alto riesgo en los factores relacionados con las demandas del trabajo, dominio que hace referencia a las exigencias que 
el trabajo le impone al individuo siendo de naturaleza cuantitativa, cognitiva, emocional, de responsabilidad, entre otras, usualmente debido al requerimiento constante de creatividad e incentivo en el trabajo, y alto grado de concentración. Estas características del trabajo docente, evaluadas como riesgo son encontradas en otras poblaciones latinoamericanas (Pando et al, 2006: 525), en las cuales también se destaca factores de riesgo como el habla y esfuerzo visual constante.

Otras variables que se encuentran en riesgo psicosocial en la población docente, son las de liderazgo y relaciones sociales en el trabajo, que hacen referencia a las relaciones que tienen los trabajadores con los superiores jerárquicos $\mathrm{y}$ colaboradores, las cuales pueden influir en la forma de trabajo y el ambiente del área de trabajo (Ministerio de la Protección Social, 2010: 20). Los resultados de estudios realizados marcaron el dominio de liderazgo y relaciones sociales, en las dimensiones de características de liderazgo y retroalimentación del desempeño, la mayoría de los docentes tuvieron un alto nivel de riesgo (Fajardo et al, 2014: 5).

También se encontró en algunas investigaciones que con respecto a la Batería de riesgo psicosocial el dominio de control sobre el trabajo, señala la percepción de niveles en riesgo medio. Aunque, se puede observar que la dimensión de claridad del rol, la cual se refiere a la forma como se da las instrucciones para desarrollar una tarea, presenta un riesgo alto y se identifica como la dimensión que más afecta la salud de los docentes (Bedoya, Oquendo \& Gallego, 2014: 108).

La presencia de factores de riesgo psicosocial mencionados anteriormente, que se encuentran presentes en la población docente generan por su parte, la sensación de inestabilidad del cargo, siendo esto la creencia a una posible pérdida del puesto de trabajo, relacionado con la modalidad de contratación en la que se encuentran, falta de reconocimiento del trabajo realizado baja retribución financiera, de estima, o de posibilidades de promoción, que obtiene el docente a cambio de su contribución o esfuerzo (Caballero et al, 2009: 150; Gómez \& Moreno, 2010: 397; Rodríguez et al, 2014: 16). En este caso se observa que el desequilibro sentido entre el esfuerzo dado por el docente y la recompensa obtenida, sea social, laboral o económica, es un fuerte factor de riesgo psicosocial.

En relación a características generales de las investigaciones encontradas en Colombia y América Latina, cabe destacar que en su mayoría, la población de estudio es reducida y seleccionadas a conveniencia de los investigadores, limitante 
que ha sido detallado en gran parte de estos estudios y el cual probablemente tiene relevancia en los resultados encontrados. Adicionalmente, la mayoría de investigaciones revisadas fueron realizadas con docentes universitarios. Por lo tanto, los estudios encontrados con docentes escolares fueron relativamente pocos, teniendo en cuenta las diferencias cualitativas existentes entre estas poblaciones, por lo que resulta importante describir los factores psicosociales en docentes escolares para que los programas seguridad y salud en el trabajo se ajusten a las necesidades de la población. Siendo así, el desarrollo de esta investigación contribuye a los docentes como colaboradores y/o trabajadores en su salud física y psíquica y al mejoramiento de su desempeño en la organización, para que esta pueda obtener un mejor funcionamiento y rentabilidad.

En consecuencia con lo mencionado anteriormente, se identifica que posiblemente la docencia se puede considerar como una profesión expuesta a múltiples exigencias dentro y fuera del lugar de trabajo que pueden desarrollar factores de riesgo psicosocial, de ahí que resulte importante la identificación de los mismos tanto a nivel intralaboral como extralaboral y a partir de esto se propone el presente estudio que tiene como objetivo la identificación de los factores psicosociales intralaborales y extralaborales, a los que se exponen los docentes de una Institución Educativa Estatal de San Juan de Pasto.

\section{MÉTODO}

El presente estudio es de tipo descriptivo, puesto que su propósito consiste en la caracterización de los factores psicosociales de la población bajo estudio. El diseño asumido es transversal, ya que la aplicación de la batería de factores de riesgo psicosociales a docentes se realizó en una sola ocasión y en un mismo periodo de tiempo (Hernández, Fernández \& Baptista, 2007: 208).

\subsection{Muestra}

De los 204 docentes pertenecientes a una institución educativa, se calculó un muestreo aleatorio simple, el cual tiene un margen de 4\% y una confianza del $96 \%$, brindando una muestra de 162 personas, que con su intención explícita por participar en el estudio proporcionaron su firma al consentimiento informado, donde se presentó el objetivo de la investigación, se especificó que ésta no genera riesgos asociados ni beneficios directos, que la identidad como participantes será 
confidencial y se mencionó, la posibilidad de abstenerse de participar o retirarse del estudio en el momento en que lo deseen (Ley 1090 de 2006).

\subsection{Procedimiento}

Se inició con la presentación del proyecto de investigación al rector de la institución educativa para solicitar el acceso a la población y organizar aspectos logísticos para la aplicación de la Batería. Posteriormente, se solicita la lista de los 204 docentes, se procede a calcular un muestreo de 162 personas las cuales se seleccionó al azar, se convocó a los docentes a participar de la investigación por medio de una socialización y sensibilización del proceso, se informó que su participación era voluntaria. Aquellos docentes que accedieron a participar de la investigación firmaron un consentimiento informado y se procedió a responder los cuestionarios.

La calificación de los cuestionarios de riesgo psicosocial intralaboral y extralaboral, tuvo en cuenta el proceso establecido por la Batería de Instrumentos para la Evaluación de Riesgos Psicosociales del Ministerio de la Protección Social (2010) que identificó el porcentaje de la población que se encuentra en los diferentes niveles de riesgo en cada una de las dimensiones al momento de la sistematización de los datos.

Para obtener los resultados se realizó la calificación que especifica la Batería, los pasos a seguir son: (1) calificación de los ítems, cada uno de los ítems del cuestionario debe ser calificado con valores (números enteros) que oscilan entre 0 y 4 .; (2) obtención de los puntajes brutos, primero se suma los ítems correspondientes por dimensión y luego el puntaje bruto de cada dominio se calcula sumando los puntajes brutos de las dimensiones que lo integran; (3) transformación de los puntajes brutos; la transformación lineal de los puntajes brutos a una escala de puntajes que van de 0 a 100. Para realizar esta transformación se utiliza la siguiente fórmula:

Puntaje transformado $=$ Puntaje bruto X 100 Factor de transformación

(4) comparación de los puntajes transformados con las tablas de baremos, comparar los puntajes transformados con las tablas de baremos, que indicarán 
el nivel de riesgo que representan las puntuaciones transformadas de las dimensiones, de los dominios y del puntaje total. Por último, se calcula el porcentaje de docentes según el nivel de riesgo (Ministerio de la Protección Social, 2010: 29-84).

\subsection{Instrumentos}

Para la medición de los factores psicosociales en los docentes, se aplicó la Batería de Instrumentos para la Evaluación de Factores de Riesgo Psicosocial elaborada por el Ministerio de la Protección Social (2010) para población colombiana. La Batería está compuesta por ocho instrumentos diferentes, de los cuales se aplicaron tres: (1) Ficha de Datos Generales, compuesto por 19 ítems, (2) Cuestionario de Riesgo Psicosocial Intralaboral (forma A) éste cuestionario va dirigido a trabajadores profesionales, está conformado por 123 ítems, su confiabilidad se encuentra estimada con Alfa de Cronbach de 0,954 y (3) Cuestionario de Riesgo Psicosocial Extralaboral que consta de 31 ítems y su confiabilidad se encuentra estimada con Alfa de Cronbach de 0,88 (Ministerio de la Protección Social, 2010: 138).

Teniendo en cuenta la definición de los factores psicosociales y la batería de riesgos psicosociales fue creada en base a la resolución 2646 de 2008 del Ministerio de Protección Social, por lo cual esta investigación se rige en la normatividad, la cual expresa que la valoración de las condiciones de trabajo y salud, se debe realizar utilizando criterios técnicos y metodológicos validados en el país, en este caso la batería de riesgos psicosociales, el cual debe ser realizado solo por profesionales en psicología con un mínimo de 100 horas de capacitación en factores psicosociales.

\subsection{Aspectos éticos y legales}

La aplicación de la Batería de Riesgos Psicosociales se realizó en el año 2016, en donde los participantes firmaron un consentimiento informado que presentó el objetivo de la investigación, se especificó que su participación en el estudio no generaba riesgos asociados ni beneficios directos, y de la misma manera, la posibilidad de abstenerse de participar o retirarse de la investigación en el momento en que lo deseen y que su identidad como participantes será confidencial. Dadas las circunstancias de confidencialidad, la institución 
solicita expresamente se reserve el nombre de la misma en el desarrollo de la investigación y publicación de los resultados.

\subsection{Resultados}

A continuación se presentan los resultados obtenidos por cada cuestionario aplicado de la Batería de Medición de Riesgos Psicosociales.

\subsubsection{Cuestionario de factores de riesgo psicosocial intralaboral (Forma A)}

A partir de los resultados encontrados en la aplicación del cuestionario se puede identificar que los docentes de la Institución Educativa municipal, I.E.M., dentro del Cuestionario de Factores de Riesgo Psicosocial Intralaboral indican que en las dimensiones de Características del liderazgo, Retroalimentación del desempeño, Claridad del rol, Capacitación, Participación y manejo del cambio, Control y autonomía sobre el trabajo, Demandas ambientales y de esfuerzo físico, Demandas emocionales, Demandas cuantitativas, Influencia del trabajo sobre el entorno extralaboral, Demandas de carga mental, Consistencia del Rol, Demandas de la jornada de trabajo y Reconocimiento y compensación, más del $40 \%$ de la población se encuentra en un riesgo alto y muy alto. Por otra parte, la dimensión de Relaciones con los colaboradores la mayor cantidad de la población presenta un riesgo bajo o despreciable, cumpliendo el papel de factor protector dentro del ambiente laboral.

A continuación, la Tabla 1 indica el porcentaje de docentes que se encuentran en cada uno de los niveles de riesgo para las dimensiones de los dominios que evalúa el Cuestionario de factores de riesgo psicosocial intralaboral (Forma A).

\section{Tabla 1}

Resultados del cuestionario de factores de riesgo psicosocial intralaboral (Forma A)

\begin{tabular}{|c|c|c|c|c|c|c|}
\hline \multirow{2}{*}{ Dominios } & Dimensiones & \multicolumn{5}{|c|}{ Nivel de riesgo } \\
\cline { 3 - 6 } & & $\begin{array}{c}\text { Sin riesgo } \\
\text { o riesgo } \\
\text { despreciable }\end{array}$ & $\begin{array}{c}\text { Riesgo } \\
\text { bajo }\end{array}$ & $\begin{array}{c}\text { Riesgo } \\
\text { medio }\end{array}$ & $\begin{array}{c}\text { Riesgo } \\
\text { alto }\end{array}$ & $\begin{array}{c}\text { Riesgo } \\
\text { muy } \\
\text { alto }\end{array}$ \\
\hline
\end{tabular}


Factores psicosociales laborales inmersos en el contexto de los docentes en una institución educativa estatal de San Juan de Pasto Dávila Burbano_Hernán Darío, Lombana Mora_Lady Dayanne, Matabanchoy Tulcan_Sonia Maritza, Zambrano Guerrero_ Christian Alexander

\begin{tabular}{|c|c|c|c|c|c|c|}
\hline \multirow{4}{*}{$\begin{array}{l}\text { Liderazgo y } \\
\text { relaciones } \\
\text { sociales en el } \\
\text { trabajo }\end{array}$} & $\begin{array}{l}\text { Características del } \\
\text { liderazgo. }\end{array}$ & $3,7 \%$ & $5,6 \%$ & $11,8 \%$ & $23,0 \%$ & $55,9 \%$ \\
\hline & $\begin{array}{l}\text { Relaciones sociales en } \\
\text { el trabajo }\end{array}$ & $8,90 \%$ & $18,30 \%$ & $29,80 \%$ & $18,80 \%$ & $24,10 \%$ \\
\hline & $\begin{array}{l}\text { Retroalimentación del } \\
\text { desempeño }\end{array}$ & $3,10 \%$ & $14,5 \%$ & $10,7 \%$ & $27,7 \%$ & $44 \%$ \\
\hline & $\begin{array}{l}\text { Relaciones con } \\
\text { los colaboradores } \\
\text { (subordinados) }\end{array}$ & $51,2 \%$ & $30,7 \%$ & $18,1 \%$ & $0,0 \%$ & $0,0 \%$ \\
\hline \multirow{5}{*}{$\begin{array}{l}\text { Control sobre } \\
\text { el trabajo }\end{array}$} & Claridad del rol & $1,9 \%$ & $9,4 \%$ & $11,3 \%$ & $34,4 \%$ & $43,1 \%$ \\
\hline & Capacitación & $5,6 \%$ & $4,9 \%$ & $19,8 \%$ & $37,7 \%$ & $32,1 \%$ \\
\hline & $\begin{array}{l}\text { Participación y } \\
\text { manejo del cambio }\end{array}$ & $2,5 \%$ & $6,2 \%$ & $14,9 \%$ & $28,0 \%$ & $48,4 \%$ \\
\hline & $\begin{array}{l}\text { Oportunidades para } \\
\text { el uso y desarrollo } \\
\text { de habilidades y } \\
\text { conocimientos }\end{array}$ & $29,8 \%$ & $18,0 \%$ & $30,4 \%$ & $11,2 \%$ & $10,6 \%$ \\
\hline & $\begin{array}{l}\text { Control y autonomía } \\
\text { sobre el trabajo }\end{array}$ & $3,8 \%$ & $13,1 \%$ & $23,8 \%$ & $33,1 \%$ & $26,3 \%$ \\
\hline \multirow[t]{8}{*}{$\begin{array}{l}\text { Demandas } \\
\text { del trabajo }\end{array}$} & $\begin{array}{l}\text { Demandas } \\
\text { ambientales y de } \\
\text { esfuerzo físico }\end{array}$ & $0,0 \%$ & $1,9 \%$ & $11,1 \%$ & $13,6 \%$ & $73,5 \%$ \\
\hline & $\begin{array}{l}\text { Demandas } \\
\text { emocionales }\end{array}$ & $2,60 \%$ & $7,30 \%$ & $11,40 \%$ & $31,60 \%$ & $47,20 \%$ \\
\hline & $\begin{array}{l}\text { Demandas } \\
\text { cuantitativas }\end{array}$ & $1,9 \%$ & $4,5 \%$ & $15,4 \%$ & $25,0 \%$ & $53,2 \%$ \\
\hline & $\begin{array}{l}\text { Influencia del trabajo } \\
\text { sobre el entorno } \\
\text { extralaboral }\end{array}$ & $0,6 \%$ & $4,3 \%$ & $14,8 \%$ & $13,0 \%$ & $67,3 \%$ \\
\hline & $\begin{array}{l}\text { Exigencias de } \\
\text { responsabilidad del } \\
\text { cargo }\end{array}$ & $9,4 \%$ & $24,4 \%$ & $35,0 \%$ & $25,0 \%$ & $6,3 \%$ \\
\hline & $\begin{array}{l}\text { Demandas de carga } \\
\text { mental }\end{array}$ & $16,8 \%$ & $13,7 \%$ & $21,1 \%$ & $22,4 \%$ & $26,1 \%$ \\
\hline & Consistencia del rol & $7,1 \%$ & $13,5 \%$ & $20,5 \%$ & $26,9 \%$ & $32,1 \%$ \\
\hline & $\begin{array}{l}\text { Demandas de la } \\
\text { jornada de trabajo }\end{array}$ & $7,0 \%$ & $10,1 \%$ & $18,4 \%$ & $20,9 \%$ & $43,7 \%$ \\
\hline
\end{tabular}




\begin{tabular}{|l|l|l|l|l|l|l|}
\hline Recompensas & $\begin{array}{l}\text { Recompensas } \\
\text { derivadas de la } \\
\text { pertinencia a la } \\
\text { organización y del } \\
\text { trabajo que se realiza }\end{array}$ & $29,4 \%$ & $17,5 \%$ & $17,5 \%$ & $16,3 \%$ & $19,4 \%$ \\
\cline { 2 - 7 } & $\begin{array}{l}\text { Reconocimiento y } \\
\text { compensación }\end{array}$ & $3,4 \%$ & $8,7 \%$ & $11,4 \%$ & $22,8 \%$ & $53,7 \%$ \\
\hline
\end{tabular}

\subsubsection{Cuestionario de factores de riesgo psicosocial extralaboral}

En el Cuestionario de factores de riesgo psicosocial extralaboral se evidencia que en las dimensiones Tiempo fuera del trabajo, Características de la Vivienda $y$ de su entorno e Influencia del entorno extralaboral sobre el trabajo, más del $40 \%$ de la población se encuentran en niveles de riesgo alto y muy alto. Finalmente, es importante mencionar que entre las condiciones extralaborales, la dimensión Relaciones Familiares, se constituye como un factor de protección, considerando el alto porcentaje de docentes se encuentran en un nivel de riesgo bajo o sin riesgo.

La Tabla 2 indica el porcentaje de docentes que se encuentran en cada uno de los niveles de riesgo para las dimensiones que evalúa el Cuestionario de factores de riesgo psicosocial extralaboral.

\section{Tabla 2}

Resultados del cuestionario de factores de riesgo psicosocial extralaboral

\begin{tabular}{|l|l|l|l|l|l|}
\hline \multirow{2}{*}{\multicolumn{1}{|c|}{ Dimensión }} & \multicolumn{5}{c|}{ Nivel de riesgo } \\
\cline { 2 - 6 } & \multicolumn{1}{|c|}{$\begin{array}{c}\text { Sin riesgo } \\
\text { o riesgo } \\
\text { despreciable }\end{array}$} & $\begin{array}{c}\text { Riesgo } \\
\text { bajo }\end{array}$ & $\begin{array}{c}\text { Riesgo } \\
\text { medio }\end{array}$ & $\begin{array}{c}\text { Riesgo } \\
\text { alto }\end{array}$ & $\begin{array}{c}\text { Riesgo } \\
\text { muy alto }\end{array}$ \\
\hline Tiempo fuera del trabajo & $4,4 \%$ & $18,4 \%$ & $13,9 \%$ & $43,0 \%$ & $20,3 \%$ \\
\hline Relaciones familiares & $50,6 \%$ & $13,3 \%$ & $13,9 \%$ & $8,2 \%$ & $13,9 \%$ \\
\hline $\begin{array}{l}\text { Comunicación y } \\
\text { relaciones interpersonales }\end{array}$ & $13,9 \%$ & $23,4 \%$ & $17,7 \%$ & $27,8 \%$ & $17,1 \%$ \\
\hline $\begin{array}{l}\text { Situación económica del } \\
\text { grupo familiar }\end{array}$ & $17,2 \%$ & $26,1 \%$ & $17,2 \%$ & $26,1 \%$ & $13,4 \%$ \\
\hline $\begin{array}{l}\text { Características de la } \\
\text { vivienda y de su entorno }\end{array}$ & $10,1 \%$ & $20,8 \%$ & $10,1 \%$ & $27,7 \%$ & $31,4 \%$ \\
\hline
\end{tabular}




\begin{tabular}{|l|l|l|l|l|l|}
\hline $\begin{array}{l}\text { Influencia del entorno } \\
\text { extralaboral sobre el } \\
\text { trabajo }\end{array}$ & $18,5 \%$ & $13,4 \%$ & $15,3 \%$ & $24,2 \%$ & $28,7 \%$ \\
\hline $\begin{array}{l}\text { Desplazamiento vivienda- } \\
\text { trabajo-vivienda }\end{array}$ & $15,7 \%$ & $18,9 \%$ & $19,5 \%$ & $23,3 \%$ & $22,6 \%$ \\
\hline
\end{tabular}

\section{CONCLUSIONES}

A partir de la bibliografía revisada en el desarrollo de la investigación se puede observar que no se registran estudios que profundicen los factores psicosociales en la población de docentes de instituciones de educación media y primaria en cuanto a la correspondencia del estudio en donde articule los factores de riesgo psicosociales intralaborales con los extralaborales, lo cual lleva a reflexionar, la importancia de continuar realizando investigaciones de este corte con esta población, dado que en los resultados encontrados se puede evidenciar que los docentes de este tipo de instituciones pueden presentar un nivel alto de riesgo psicosocial intralaboral como extralaboral reflejándose en la salud y seguridad en el trabajo, lo que puede afectar la labor desempeñada y posiblemente alterar el funcionamiento adecuado de la institución referente a lo administrativo y formación académica impartida a niños y adolescentes.

Teniendo en cuenta los resultados que se presentan en el cuestionario de factores de riesgo psicosocial intralaboral (forma $\mathrm{A}$ ), se reconoce que en la dimensión de características del liderazgo relacionada con interacción con los jefes inmediatos, la mayor parte de la población se encuentra en riesgo alto y muy alto, lo que puede significar que existe una percepción de dificultades para comunicarse eficientemente, donde el apoyo social del jefe es escaso o nulo, de igual forma lleva también a percibir que la gestión realizada por el jefe se visibilice con dificultades en la planificación (Ministerio de la Protección Social, 2010: 24). Los resultados también demuestran que quienes ejercen roles de dirección pueden llegar a sentir, en un nivel considerable, que no están respaldados por sus trabajadores, evidencia de una relación vertical, lo que implica una comunicación de arriba hacia abajo, donde hay poca colaboración entre las partes, que se refleja en la falta de retroalimentación positiva del jefe hacia sus colaboradores y viceversa. 
En el dominio de relaciones sociales en el trabajo se observa que su punto más alto está en riesgo medio con una tendencia a incrementar, lo cual representa que los docentes perciben que existen dificultades en la integración, interacción y apoyo con sus compañeros de trabajo, además de la posibilidad de generar pocos espacios para el desarrollo de trabajo en equipo. Lo cual es generado por cierto tipo de sectorización que se establece dentro de la institución entre los colaboradores al momento de generarse pequeños grupos identificados por sus áreas de aplicación, lo que conlleva a un auto enriquecimiento o desarrollo del grupo más no de la institución como una organización.

La Retroalimentación del desempeño es una condición muy importante para el crecimiento no solo personal, sino institucional. En este caso se puede evidenciar que es percibida en un riesgo alto y muy alto, lo que concluye que los docentes no reciben una adecuada retroalimentación de su labor como tal, ya sea para mantener sus fortalezas o poder implementar un plan de mejoramiento en cuanto a sus debilidades. Este tipo de procesos que ayuden al mejoramiento del individuo son muy importantes y fundamentales y más aún si es en una organización grande como los colegios, ya que el beneficiado no solo sería quien recibe la retroalimentación sino también los directivos por tener un personal que va a aportar más a un buen funcionamiento de su institución, y un usuario en este caso los estudiantes que van a recibir de mejor manera o de mayor calidad un derecho que se establece como servicio, en este caso su formación académica.

En cuanto a la Relación con los colaboradores (subordinados) puede percibirse como riesgo por los docentes ya que pueden existir dificultades para comunicarse respetuosamente, además pueden sentir que los jefes no permiten o fomentan la participación en las decisiones importantes, generando que los docentes crean que su labor y presencia en el lugar de trabajo no es reconocida (Ministerio de la Protección Social, 2010: 24). Teniendo en cuenta que las relaciones en el lugar de trabajo son fundamentales y que de estas depende el buen funcionamiento tanto de la institución como el bienestar de todos los trabajadores se ve necesario ampliar la información para poder generar estrategias que mejoren las relaciones entre directivos y colaboradores.

Teniendo en cuenta el dominio control sobre el trabajo, más de la mitad de la población se encuentra en nivel de riesgo alto y muy alto, evidenciando 
una diferencia significativa en comparación a los resultados de los estudios realizados por Rodríguez, et al, (2014: 4) en el cual se evidencia que la mayor parte de docentes presentan un nivel de riesgo bajo y riesgo despreciable, siendo uno de los que menos nivel de riesgo tiene en ese estudio. De acuerdo a lo anterior, se puede concluir que los docentes de colegios perciben que tienen baja participación y pocas posibilidades de influir sobre su trabajo, esto posiblemente a la reducida flexibilidad que tienen en el manejo de los contenidos y de horarios ya que la diferencia con los docentes universitarios, se enfatiza en la autonomía y posibilidad de desarrollar flexiblemente su cátedra. Ante esto, los profesores pueden llegar a enfrentarse a un sentimiento de frustración al no poder desarrollar sus potencialidades como docentes, siendo restringidos por múltiples factores como horarios, objetivos curriculares, número de estudiantes, procesos de evaluación docente, entre otros. Lo cual puede ser reflejo de inseguridad que sienten al enfrentarse a su rol de educador, e inseguridad al tener que cumplir con múltiples expectativas que difieren de su rol como son las expectativas de la política educativa del gobierno, de los directivos, de los padres de familia, de los estudiantes y de sus mismos compañeros educadores.

Respecto a la dimensión de capacitación, se percibe en riesgo alto y muy alto, ya que los docentes denotan que posiblemente las capacitaciones no tienen las características necesarias o no responden a las necesidades concretas para el desarrollo y fortalecimiento de sus conocimientos y habilidades para aportarle de mejor manera al crecimiento de su institución y a la adecuada formación académica de los estudiantes.

En el dominio demandas de trabajo, el presente estudio arrojó resultados con nivel de riesgo muy alto en la dimensión demandas ambientales y de esfuerzo físico en la mayor parte de la población, al contrastar con los resultados de "Factores de Riesgo Psicosocial Intralaboral y grado de Estrés en docentes universitarios" Rodríguez et al, (2014) se observa la gran diferencia, ya que presenta un nivel despreciable de riesgo en más de la mitad de la población. Por lo cual, se puede concluir que los múltiples escenarios que se brindan en las instituciones y las organizaciones generan diferentes condiciones laborales, las cuales influyen en el rol del docente, entre estas condiciones se reconoce las distintas exigencias y esfuerzos en cuanto a cantidad, que se presentan en cada organización, esto posiblemente a que los docentes de colegios atienden 
un mayor número de población ya que no solo se encargan de los estudiantes y tareas administrativas, sino también de atender a los padres de familia y brindar espacios para responder a las diferentes problemáticas presentes en la institución.

La institución educativa es un lugar donde el profesor está expuesto a múltiples situaciones a las cuales tiene que responder de forma inmediata, esto hace que deba estar atento y pendiente todo el tiempo, por lo cual tiene pocos espacios para descansar y relacionarse con sus colegas por razones distintas a las meramente laborales. Dentro de éste también se puede evidenciar la carga de trabajo que los docentes tienen que llevar a su entorno extralaboral como lo es su espacio personal y/o familiar, invadiendo su privacidad y afectando las relaciones personales en cuanto a la calidad de tiempo que comparte con los suyos.

Dentro del análisis que se realiza del dominio de recompensas, donde las dimensiones de recompensas derivadas de la pertinencia a la organización y del trabajo que se realiza, reconocimiento y compensación se encuentran en riesgo alto y muy alto, refiere que el docente dentro de su labor diaria no se siente retribuido adecuadamente en cuanto al desarrollo y desempeño de su labor. Acorde a lo anterior se observa que los docentes perciben que no tienen el reconocimiento laboral y social el cual es brindado por los mismos estudiantes que son quienes reciben directamente el beneficio de su conocimiento, como tampoco el de sus pares o compañeros de trabajo y de los administrativos y jefes que acompañan en el desarrollo diario de la organización. En cuanto a la compensación monetaria, los docentes no se ven reconocidos y valorados por los entes gubernamentales ya que no les brindan garantías en cuanto a un contrato adecuado con una remuneración pertinente en comparación al papel desempeñado. Lo que conlleva a un desequilibrio entre el esfuerzo que el docente realiza a la recompensa o contribución recibida ya sea social, laboral o económica (Caballero et al, 2009: 150; Gómez \& Moreno, 2010: 397; Rodríguez et al, 2014: 16).

En las dimensiones que componen el cuestionario de factores de riesgo psicosocial extralaboral, se encuentran tiempo fuera del trabajo con más de la mitad de la población en un riesgo alto y muy alto, donde se evidencia que los docentes perciben que el tiempo que destinan para su descanso o de compartir 
con su grupo familiar y/o social es limitante, interfiriendo e invadiendo con lo laboral su espacio personal como lo es el dormitorio, espacio de comedor y demás espacios que deberían tener un fin único en cuanto a su uso. Esto puede deberse a la gran carga laboral y también a la forma en como los docentes administran el tiempo en su jornada laboral, lo cual causa que lleven trabajo a sus hogares, generando dificultades en las relaciones familiares por el escaso tiempo que le pueden dedicar a los miembros de su familia, lo que puede ocasionar el rompimiento de los vínculos familiares y sociales influyendo también en la adecuada labor de los docentes en las instituciones.

En cuanto a las características de la vivienda y de su entorno es percibida en riesgo alto y muy alto ya que los docentes consideran que las condiciones de su vivienda y su entorno no son los adecuados para residir, por las diferentes comodidades que son necesarias para realizar el trabajo que se llevan a sus hogares como también para un adecuado descanso y movilidad al lugar de trabajo ya que esto puede generar costos tanto monetarios como físicos.

Teniendo en cuenta lo anterior, se puede observar la influencia del entorno extralaboral sobre el trabajo, en donde la vida personal de los docentes posiblemente se ve inmersa en la funcionalidad de su labor y el bienestar propio, y desplazamiento vivienda-trabajo-vivienda, juega un papel importante en cuanto al acceso que tienen para poderse transportar los docentes a sus sitios de trabajo, estas son percibidas como un factor de riesgo ya que son condiciones que si influyen en la salud, bienestar del trabajador lo cual interfiere en la labor que tienen en su organización, no siempre tiene una intervención inmediata para poderlo solventar como factor de riesgo, ya que muchas veces depende del factor económico por el cual se ven afectados al no tener el reconocimiento (Ministerio de la Protección Social, 2010: 26) por medio de un contrato adecuado que brinde un salario acorde a su funcionalidad e importancia como trabajadores de la educación.

Los resultados que brinda el cuestionario de factores de riesgo psicosocial extralaboral, demuestra que los docentes se encuentran expuestos a diferentes condiciones que afectan su salud, tanto dentroy fuera de su trabajolas cuales afectan su desempeño en la organización, entre estas condiciones se puede encontrar las relaciones con los familiares, el lugar de residencia, el desplazamiento al lugar del trabajo y otras condiciones que generan malestar y afectan el bienestar de los 
docentes. Es por ello que se reconoce la importancia de determinar los factores de riesgo extralaborales, ya que no hay una profundización en la investigación del mismo, siendo esto, importante para la promoción y prevención de riesgos que afecten la vida laboral y personal de los docentes y de los demás trabajadores que integran cada una de las organizaciones existentes.

\section{RECOMENDACIONES}

Se hace necesario un estudio más amplio con la población de docentes de instituciones educativas de básica primaria, básica secundaria y educación media, tanto en el municipio de Pasto, como en otros municipios de los diferentes departamentos, ya que es una problemática que afecta no solo la calidad de vida de los trabajadores sino también su rendimiento laboral y el de la organización.

Es importante desarrollar un programa de prevención de factores de riesgo psicosocial intralaboral y extralaboral que permitan el mejoramiento de la calidad de vida del docente.

Proponer la creación de programas de intervención para tratar los riesgos intralaborales y extralaborales, por medio de un trabajo interdisciplinar que permita el mejoramiento del desarrollo personal y organizacional.

Se debe implementar el desarrollo de programas preventivos del manejo del estrés laboral, con miras al sostenimiento y promoción de la calidad de vida de los docentes lo cual lo puede desarrollar la institución por medio de la entidad de salud que la precede o los entes de gobierno pertinentes.

Para el adecuado mejoramiento y funcionamiento las instituciones $\mathrm{y}$ las diferentes organizaciones que se ven afectadas por los factores de riesgo psicosocial, se podría tener en cuenta el concepto de solidaridad orgánica realizado por Durkheim (Lucas, 1993 citado en Duque, 2013: 194), que determina la interdependencia de los individuos, vinculado cada uno de estos hacia una cooperación del grupo, en donde exige la coordinación entre los diferentes órganos que constituyen la organización en donde se tome una posición activa y positiva frente a las dificultades que atraviese el trabajador, el empleador y la organización en general, lo que contribuiría positivamente en un interés colectivo y así la organización pueda obtener una mayor rentabilidad en su objetividad. 


\section{REFERENCIAS}

(1) Bedoya, Bedoya Ever de Jesús., Oquendo, Puerta Sergio René. \& Gallego, Ruiz María Camila. (2014). Diagnóstico de los factores de riesgos psicosociales intralaborales a los que están expuestos los docentes de tiempo completo y de cátedra en la Universidad Autónoma Latinoamericana (UNAULA). Revista Uni-pluri/versidad 14 (3), 102-113.

(2) Botero, Álvarez Carla. (2012). Riesgo psicosocial intralaboral y "burnout" en docentes universitarios de algunos países latinoamericanos. Cuadernos de Administración, 28 (48), 117-132.

(3) Caballero, Dominguez Carmen., González, Gutierres Orlando., Mercado, Anguila Daniel., Llanos, Barrios Carlos., Bermejo, Barrero Yamid. \& Vergel, Medrano Claudia. (2009). Prevalencia del síndrome de burnout y su correlación con factores psicosociales en docentes de una institución universitaria privada de la ciudad de Barranquilla. Revista Psicogente, $12(21), 142-157$.

(4) CLadellas, Pros Ramón. \& Badia, Martin María del Mar. (2010). La gestión del tiempo de los profesores universitarios en función de la modalidad educativa: sus efectos psicosociales. Revista Española de pedagogía, LXVIII (246), 297-310.

(5) Comité Mixto OIT/OMS. (1984). "Factores psicosociales en el trabajo: naturaleza, incidencia y prevención”. Novena reunión. Ginebra, 1. Serie seguridad, higiene y medicina del trabajo núm. 56.

(6) Duque, María Paula. (2013). El concepto de solidaridad. Revista de Estudios Sociales, (46), 192-194.

(7) EnríqueZ, Villota María Fernanda. (2008). Factores de Riesgo Psicosocial en docentes de la Universidad Mariana. Revista UNIMAR, 45, 73-87.

(8) Fajardo, Zapata Álvaro., Montejo, Ángel Fernando., Molano, Vargas German., Hernández, Niño Jenny. \& Quintero, Guzmán Adelaida. (2013). Correlación entre los Factores de Riesgo Intralaboral y los Niveles Séricos de Cortisol en Docentes Universitarios. Ciencia y Trabajo, 15 (46), 1-6.

(9) Gómez, Ortiz Viviola. \& Moreno, Lorena. (2010). Factores psicosociales del trabajo (demanda-control y desbalance esfuerzo-recompensa), salud mental y tensión arterial: un estudio con maestros escolares en Bogotá, Colombia. UniversitasPsychologica, 9 (2), 393407.

(10) Herazo, Ruiz Cristian Camilo, OSPINA, Serna Michelle \& Ramirez, Henao Sandra. (2013). Factores psicosociales y organizacionales que afectan la productividad. Trabajo de 
grado presentado como requisito para optar el Título de Especialista en Gestión del Talento Humano y la Productividad, Universidad de Medellín, Medellín, Colombia.

(11) Hernández, Sampieri Roberto., Fernández, Collado Carlos., \& Baptista, Lucio María del Pilar. (2007). Metodología de la investigación. (4a . ed.). México: McGraw Hill.

(12) Ministerio de la Protección Social, (2008). Resolución 2646. Colombia.

(13) Ministerio de la Protección Social. (2010). Batería de Instrumentos para la Evaluación de Factores de Riesgo Psicosocial. Colombia.

(14) Múnera, Palacio Mónica María., Sáenz, Agudelo Mónica Liliana., Cardona, Arango Doris. (2013). Nivel de riesgo psicosocial intralaboral de los docentes de la Facultad de Medicina, Universidad CES Medellín, 2011. Rev. CES Med., 27 (2) ,163-175.

(15) Organización Internacional del Trabajo. (2002). Registro y notificación de accidentes del trabajo y enfermedades profesionales y lista de la OIT relativa a las enfermedades profesionales. Obtenido de, http://www.ilo.org/public/spanish/standards/relm/ilc/ilc90/rep-v-1. htm\#Registro\%20y\%20notificacion\%20de\%20accidentes\%20deL \%20traba

(16) Pando, Moreno Manuel., Castañeda, Torres Jorge., Gregoris, Gómez Martín., Águila, Marín Antonio., Ocampo, De Águila Lourdes. \& Navarrete, Rosa María. (2006). Factores psicosociales y síndrome de burnout en docentes de la Universidad del Valle de Atemajac, Guadalajara, México. Salud en Tabasco, 12 (3), 523-259.

(17) Peláez, Arcila Sandra Milena. \& Cuellar, García Susana. (2014). Identificación de los factores de riesgo psicosociales, intralaborales, en los trabajadores de la fundación Sofía, de la ciudad de Manizales. Tesis de especialización no publicada, Universidad de Manizales, Manizales, Colombia.

(18) Pérez, Soriano Javier. (2009). Seguridad y salud en los docentes. Gestión práctica de riesgos laborales: Integración y desarrollo de la gestión de la prevención, (58), 30- 35.

(19) Puentes, Suarez Alexandra., Hernández, Delgadillo Victoria. \& Sánchez, Huertas Natalia. (2008). Factores de Riesgo Psicosocial en docentes de básica primaria de Tunja. Perspectiva Salud-Enfermedad, 14 (1), 16-34.

(20) Rodríguez, Eduar., Sánchez, Gómez Johana., Dorado, Hugo Armando. \& Ramírez, José Manuel. (2014). Factores de Riesgo Psicosocial Intralaboral y grado de Estrés en docentes universitarios. Revista Colombiana de Salud Ocupacional, 4 (2), 12-17.

(21) Rojas Gutiérrez Renán Alfonso. (2015). La Seguridad y Salud en el Trabajo en Cifras. Consejo Colombiano de Seguridad. Obtenido de https://ccs.org.co/salaprensa/index.php?option $=$ com_content\&view $=$ article $\& i d=573:$ sst\&catid $=320 \&$ Itemid $=856$ 
(22) Sauter, Steven., Murphy, Lawrence., Hurrell, Joseph., \& Levi, Lennard. (2001). Factores psicosociales y de organización. En J. Stellman\& M. McCann. Enciclopedia de Salud y seguridad en el trabajo (pp.34.1-34.87). España: Chantal Dufresne, BA.

(23) Velásquez, Arboleda Oscar Hernán. \& Bedoya, Bedoya Ever de Jesús. (2010). Una aproximación a los factores de riesgo psicosocial a los que están expuestos los docentes contratados bajo la modalidad de horas cátedra en la ciudad de Medellín. UNI PLURI/VERSIDAD, $10(2), 1-14$.

(24) Vélez, Laguado Patricia. \& Mayorga, Ayala Luz Dary. (2013). Relación de los factores de riesgo psicosociales y el síndrome de burnout en docentes de planta de la Universidad Francisco de Paula Santander en el periodo del 2011-2012. Revista ciencia y cuidado, 10 (2), 85-95. 